\title{
The Fastest Recovery of Two Competing Stocks
}

\author{
Yasushi Harada* and Kazumi Sakuramoto* \\ (Received May 8, 1992)
}

\begin{abstract}
The stocks of economically valuable fish species are easily depleted, because they are selectively harvested. Although the importance of their recovery is obvious from both conservational and economical viewpoints, it is usually difficult because their intrinsic growth rates are low. Competition with species with a larger intrinsic growth rate may further increase the difficulty. In this paper, the fastest way to attain a predetermined target stock level by controlling the harvest is obtained for a two-species competition system. The result indicates that, in order to attain the target rapidly, the stock with the larger intrinsic growth rate should first be harvested intensively and the relative abundance of it should be reduced below that at the target. A suboptimal but practical procedure, which is more acceptable than the optimal one when considering the conservational point of view, is also proposed, and its performance is compared with that of a more conservational procedure.
\end{abstract}

In multi-species fisheries, the stock of the more valuable species is usually depleted quickly, because it is selectively harvested. For example, large baleen whale species such as blue and fin whales have been selectively harvested under the BWU (blue whale unit) catch quota system of the IWC, and their abundance was severely reduced. Arnalogous situations exist in other fields of fisheries on multiple stocks: an example is the trawl fishery in the East China Sea. ${ }^{1)}$ Further, the stock of the valuable species may be easily depleted even if there is no selective harvesting, because it usually has a smaller intrinsic growth rate than those coexisting.

In the discussion of the management of Antarctic minke whale stocks, it is sometimes argued that competition for food, Antarctic krill $E u$ phausia superba, between this species and blue, fin, humpback, and sei whales should be considered. ${ }^{2}$ Although the assumption of interspecific competition is still arguable, ${ }^{2,3)}$ it is important to study its possible implication on the management of the baleen whale species. ${ }^{3,4}$

In this paper, we analyze a dynamical system model of two competing stocks under harvesting and obtain the fastest way to attain the predetermined target stock level by controlling the harvest (for an introduction to the dynamical system theory on which the analysis in this paper is based, see, for example, Clark ${ }^{5}$ ). We also suggest a suboptimal but practical procedure that is more acceptable than the optimal one when considering the conservational point of view, and, by using a numerical example, compare its performance with that of a more conservational procedure.

\section{The Fastest Procedure}

Suppose that the abundance of two competing stocks, $x$ and $y$, are governed by a system of differential equations,

$$
\left\{\begin{array}{l}
\frac{\mathrm{d} x}{\mathrm{~d} t}=x f(x, y)-h_{x}, \\
\frac{\mathrm{d} y}{\mathrm{~d} t}=y g(x, y)-h_{y},
\end{array}\right.
$$

where $h_{x}$ and $h_{v}$ are instantaneous harvesting rates of $x$ and $y$, respectively. Competition between two stocks implies that per capita natural growth rates of $x$ and $y$ ( $f$ and $g$, respectively) are decreasing functions of the other stock's abundance: $i . e . \partial f / \partial y<0$ and $\partial g / \partial x<0$. The problem considered in this paper is "How should we control $h$ 's $(\geq 0)$ in order to take $x$ and $y$ from the initial stock level, $\mathrm{A}\left(x_{0}, y_{0}\right)$, to the target, B $\left(x_{\infty}, y_{\infty}\right)$, as fast as possible?'. We here consider the case in which $x_{\infty}<x^{*}$ and $y_{\infty}<y^{*}$, where $\left(x^{*}, y^{*}\right)$ is called "virgin stock level" and is the equilibrium abundance under no harvesting. We assume that $f$ and $g$ are positive if both $0 \leq$ $x \leq x_{\infty}$ and $0 \leq y \leq y_{\infty}$ are satisfied. A sufficient condition for this is $\partial f / \partial x<0$ and $\partial g / \partial y<0$, in other words, the absence of self-depensation in the growth rate, such as an Allee effect. We disregard

* Department of Fisheries Resources Management, Tokyo University of Fisheries, Konan, Minato, Tokyo 108, Japan (原田泰志，桜本和美：東京水産大学資源管理学科) 


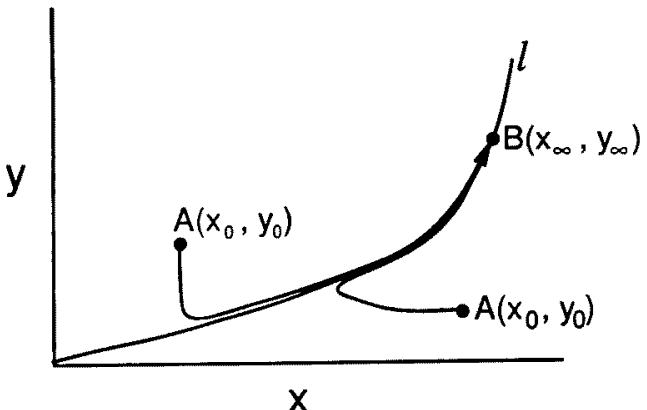

Fig. 1. The fastest approach path from below and above $l$.

cases in which $x_{0}>x_{\infty}$ and $y_{0}>y_{\infty}$, because, in such cases, we simply should harvest the excess as rapidly as possible.

Let us denote by $l$ the trajectory of the noharvesting system

$$
\left\{\begin{array}{l}
\frac{\mathrm{d} x}{\mathrm{~d} t}=x f(x, y), \\
\frac{\mathrm{d} y}{\mathrm{~d} t}=y g(x, y),
\end{array}\right.
$$

that passes B. The procedure for the fastest attainment is proved to be "If $A$ is below $l$, harvest $x$ as rapidly as possible and take the stock level on $l$, then stop harvesting and wait until B is reached. If $\mathrm{A}$ is above $l$, harvest $y$ as rapidly as possible and take the stock level on $l$, then stop harvesting and wait until B is reached" (Fig. 1). In mathematical terms, this procedure is expressed as

and

$$
h_{y}=\left\{\begin{array}{lll}
h_{y}^{\max } & \text { if } & y>y_{l}(x), \\
0 & \text { if } & y \leq y_{l}(x),
\end{array}\right.
$$

$$
h_{x}=\left\{\begin{array}{lll}
h_{x}^{\max } & \text { if } & x>x_{1}(y), \\
0 & \text { if } & x \leq x_{2}(y),
\end{array}\right.
$$

where $h_{x}^{\max }$ and $h_{y}^{\max }$ are the largest possible harvesting rate of $x$ and $y$, respectively, and $l$ is denoted by $y=y_{2}(x)$ or $x=x_{l}(y)$. Hereafter, we denote the resulting most rapid path by $c$.

The optimality of $c$ can be proved in two steps as follows. We first show that the fastest path connecting two points on $l$ is $l$ itself (Fig. 2a). Because $f$ is a decreasing function of $y, \mathrm{~d} x / \mathrm{d} t$ is smaller at a point above $l$ than at a point on $l$ with the same $x$. Therefore, it takes more time to gain the difference in $x$ between the two points if we follow a path $l_{1}$ which is above $l$. Similarly, we can show that it takes more time to gain the difference in $y$ between the two points if we follow a path below $l$. Therefore, we should stop harvesting and follow $l$ once the stock level is

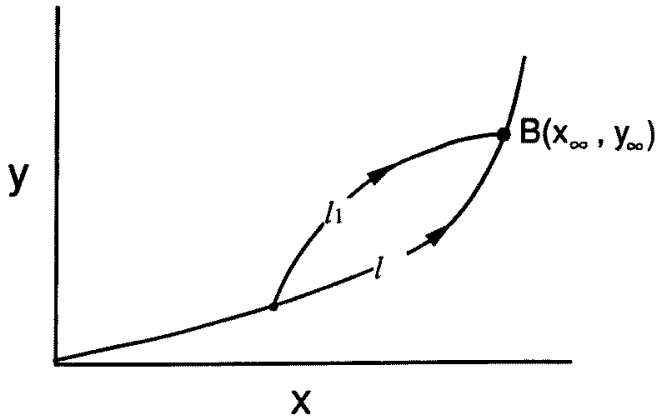

(a)

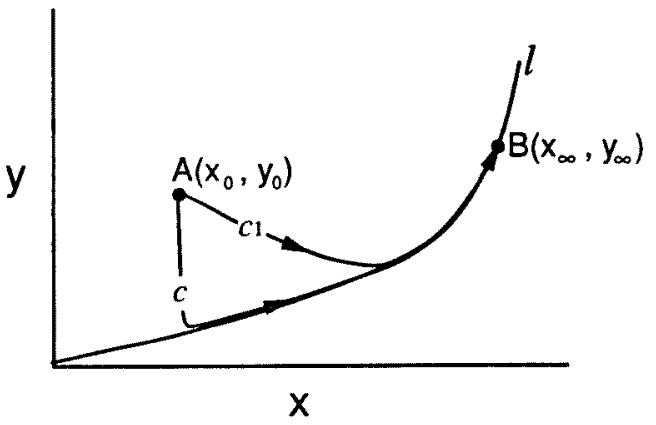

(b)

Fig. 2. Figures used in the proof of optimality of the proposed procedure. See main text for explanation.

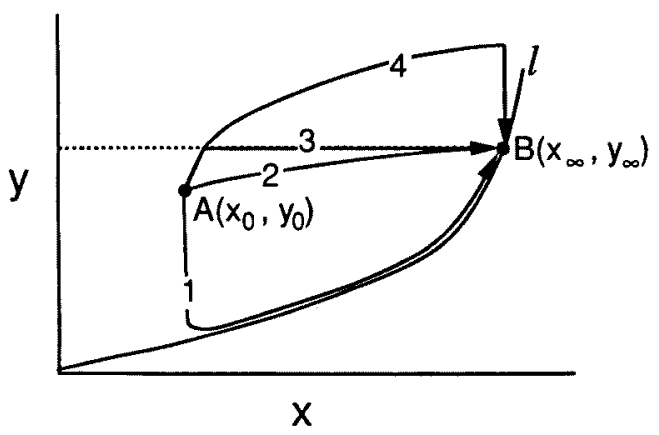

Fig. 3. Four possible trajectories realized by harvesting $y$. 1) by the most rapid procedure, 2) by constantly harvesting $y, 3$ ) by harvesting $y$ after it reaches its target level and keeping it constant, and 4) by not harvesing $y$ until $x$ reaches its target value.

on $l$. In other words, the optimal path does not cross $l$. Let us denote by $\mathbf{S}$ the set of possible trajectories that connect two points, A above $l$ and $\mathrm{B}$, and do not cross $l$. We now show that the fastest path in $\mathrm{S}$ is $c$ (Fig. 2b). The fact that $h_{x}=0$ on $c$ and $\partial f / \partial y<0$ implies that, for a value of $x$, $\mathrm{d} x / \mathrm{d} t$ takes the larger value at the point on $c$ 
than that on a path $c_{1}$ above $c$. Therefore, the difference in $x$ between $\mathbf{A}$ and $\mathbf{B}$ is gained most rapidly by following $c$. Thus, if $\mathrm{A}$ is above $l$, $B$ can be attained most rapidly by the proposed procedure. We can also show the optimality of the proposed procedure for $A$ below $l$, by using the monotonousness of $g$ as a function of $x$. Similarly, we can show that four possible trajectories shown in Fig. 3, that can be realized by controlling the harvest of $y$, are graded by the time needed to attain the target as numbered. Therefore, the most conservational strategy, 4, is the slowest one.

\section{Case of the Lotka-Volterra Competition System}

The no-harvesting trajectory, $l$, for the LotkaVolterra competition system

$$
\left\{\begin{array}{l}
\frac{\mathrm{d} x}{\mathrm{~d} t}=r_{x}(1-a x-b y) x-h_{x}, \\
\frac{\mathrm{d} y}{\mathrm{~d} t}=r_{y}(1-c x-d y) y-h_{y},
\end{array}\right.
$$

is shown in Fig. 4, for various ratios of $r_{x}$ to $r_{y}\left(r_{x} \leq r_{y}\right)$, with $a=d=1$ and $b=c=0.5$. The target is set at a point on which both stocks are reduced by the same fraction of their virgin level: i.e. $\left(x_{\infty}, y_{\infty}\right)=3 / 4\left(x^{*}, y^{*}\right)$. Fig. 4 indicates that the relative abundance of the stock with the larger intrinsic natural growth rate, $y / x$, should be reduced below that at the target, $y_{\infty} / x_{\infty}$, and it should be reduced more as the ratio of $r_{x}$ to $r_{y}$ becomes

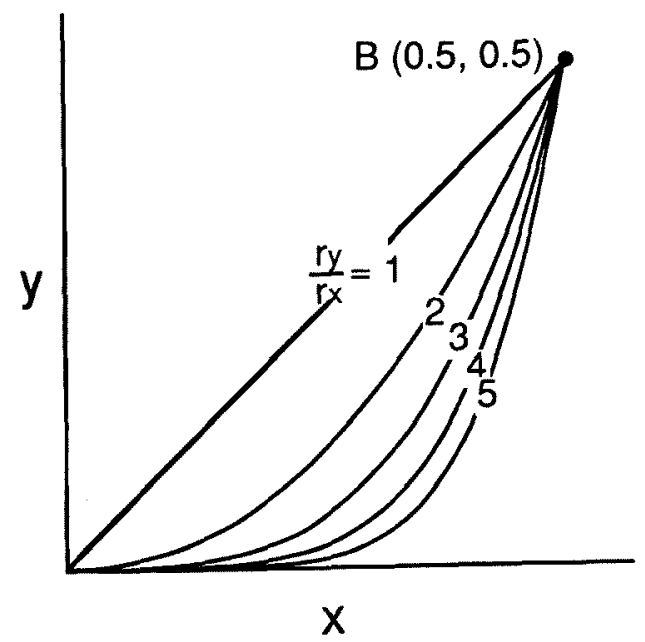

Fig. 4. $l$ for various ratios of $r_{v}$ to $r_{x}$. Target is B $(0.5,0.5)$, or $3 / 4$ of virgin stock level. larger. The same result holds in general LotkaVolterra competition systems of two stocks that have a stable coexisting equilibrium under no harvesting, if the target is set so that the relative abundance of the stock with the smaller intrinsic growth rate is not smaller than that at their virgin level (see Appendix 1 for proof).

Considering that the species with the lower intrinsic growth rate usually has higher commercial value and is expected to be more severely depleted, it can be concluded that very severe suppression of the currently more abundant stock is needed in order to recover the currently less abundant stock as rapidly as possible.

\section{A Practical Procedure}

The fastest approach path to the target obtained in the previous section requires information of $l$ and involves severe suppression of the stock with the larger intrinsic growth rate, which may result in the extinction of the stock if there is uncertainty in the population dynamics due to an estimation error or environmental variation. Therefore, it may be unacceptable from the conservational point of view, although it facilitates the recovery of the currently depressed stock. It may be unacceptable also from the economical point of view, because it requires closing of fisheries for some length of time. Thus, we here suggest a more practical procedure that is more conservational for the stock with the larger intrinsic growth rate. The procedure is described as "Harvest the stock with the larger intrinsic growth rate as fast as possible until the relative abundance reaches its final value, $y_{\infty} / x_{\infty}$, and then keep the relative abundance at $y_{\infty} / x_{\infty}$ until the target is attained". This can be written in mathematical terms as

$$
h_{x}= \begin{cases}h_{x}^{\max } & \text { if } \frac{y}{x}<\frac{y_{\infty}}{x_{\infty}}, \\ 0 & \text { if } \frac{y}{x}>\frac{y_{\infty}}{x_{\infty}},\end{cases}
$$

and similar for $h_{y}$. When $y / x=y_{\infty} / x_{\infty}$, the stock with the larger intrinsic growth rate should be harvested to keep the relative abundance at $y_{\infty} / x_{\infty}$. The procedure is faster than those described by 2,3 , and 4 in Fig. 3.

Here we compare the performance of this procedure with that of a more conservational one. Let us consider the system (2) with $a=d$ and $b=c$, 


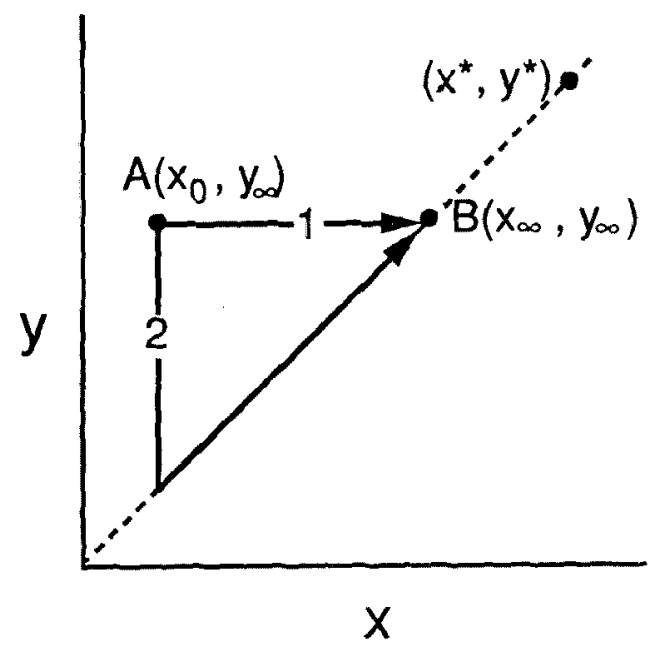

Fig. 5. Two paths considered in the numerical example. $\left(x_{\infty}, y_{\infty}\right)$ is $k\left(x^{*}, y^{*}\right)$, where $0<k<1$, and $\left(x_{0}, y_{0}\right)=\left(x_{0,}, y_{\infty}\right)$.

$$
\left\{\begin{array}{l}
\frac{\mathrm{d} x}{\mathrm{~d} t}=r_{x}(1-a x-b y) x-h_{x} \\
\frac{\mathrm{d} y}{\mathrm{~d} t}=r_{y}(1-b x-a y) y-h_{y}
\end{array}\right.
$$

in which $r_{x} \leq r_{y}$ and $a>b$, and compare the time needed to attain the target, $B\left(x_{\infty}, y_{\infty}\right)=\left(k x^{*}\right.$, $\left.k y^{*}\right)$, from A $\left(x_{0}, y_{\infty}\right)$ by each of the two paths shown in Fig. 5.

Path 1 is realized by a procedure that does not reduce $y$ below its target level defined by

$$
h_{x}=0
$$

and

$$
h_{y}=r_{y}\left(1-b x-a y_{\infty}\right) y_{\infty} .
$$

Path 2 is realized by our practical procedure with $h_{y}^{\max }=\infty$ : after the relative abundance reaches its final value,

$$
h_{x}=0
$$

and

$$
h_{y}=\frac{1}{a}\left(r_{y}-r_{x}\right)\left\{1-\left(1+\frac{b}{a}\right) x\right\} x .
$$

As shown in Appendix 2, it takes

$$
T_{1}=\frac{1}{r_{x}\left(1-b x_{\infty}\right)}\left[\log \frac{x_{\infty}}{x_{0}}-\log \frac{(a+b) x_{\infty}-1}{\left(a x_{0}+b x_{\infty}-1\right)}\right]
$$

by path 1 , and

$$
T_{\mathrm{s}}=\frac{1}{r_{x}}\left[\log \frac{x_{\infty}}{x_{0}}-\log \frac{(a+b) x_{\infty}-1}{(a+b) x_{0}-1}\right]
$$

by path 2 . In Fig. 6, we show $T_{1}, T_{2}$, and $T_{1} / T_{2}$ as a function of the relative intensity of interspecific competition and the target stock level. From these figures, we can see that the difference between $T_{1}$ and $T_{2}$ is large when $k\left(=x_{\infty} / x^{*}\right)$ and $b / a$ are large. In other words, the merit of choosing path 2 is large when the relative intensity of interspecific competition is high and the high target level is selected, although it is not very large unless both of them are very high.

\section{Discussion}

Clark $^{\text {s) }}$ considered an optimal harvesting strategy that maximizes the discounted sum of the economic benefit from the system of interacting stocks. He obtained the optimal equilibrium stock level, $\left(x_{\text {opt }}, y_{\text {opt }}\right)$, but did not obtain the optimal path to the equilibrium. He suggested the following approach rule:

$$
h_{x}=\left\{\begin{array}{lll}
h_{x}^{\mathrm{max}} & \text { if } & x>x_{\mathrm{opt}} \\
0 & \text { if } & x<x_{\mathrm{opt}}
\end{array}\right.
$$

and similar for $h_{y}$. This corresponds to path 3 in Fig. 3 and path 1 in Fig. 5.

In this paper, we set the objective of approaching the predetermined target as rapidly as possible. of course, this objective will not be compatible with the maximization of economic benefit, even when the target is set at $\left(x_{\text {opt }}, y_{\text {opt }}\right)$. But the fastest approach can be an objective when we consider the conservation of the species endangered due to overharvesting as well.

In the two species Lotka-Volterra competition system with symmetric competition coefficients, the sum of the sustainable production of two species is maximized when the levels of both species are kept at half their virgin levels. ${ }^{g)}$ As the results of this paper indicate, the stock of the species with the larger intrinsic growth rate should first be reduced below half its virgin level to attain such a two-species MSYL (maximum sustainable yield level) as rapidly as possible.

The fastest path requires severe suppression of the species with the larger intrinsic growth rate, and, to determine this, reliable information on population dynamics is needed. If there is a large error in the estimation of the population dynamics or a large environmental variation, accidental suppression below the desirable level may result in the extinction of the species. Even if it does not lead to extinction, it will slow the recovery process. Thus we proposed a suboptimal procedure which requires no information 


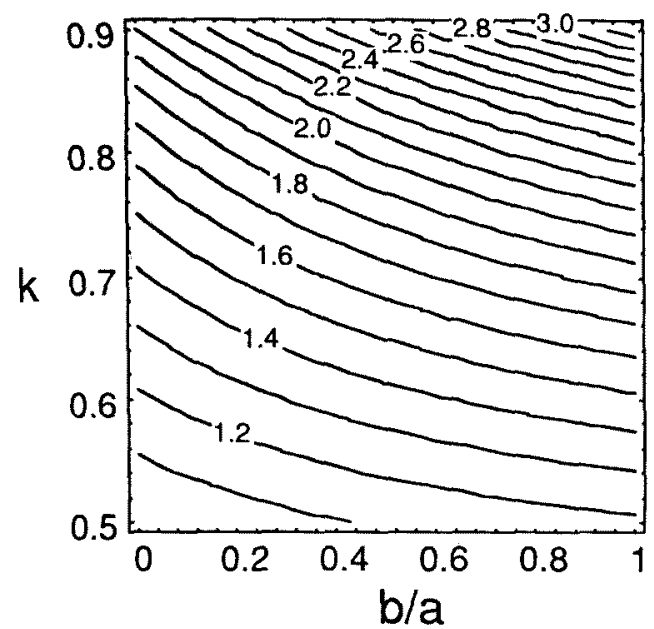

(a)

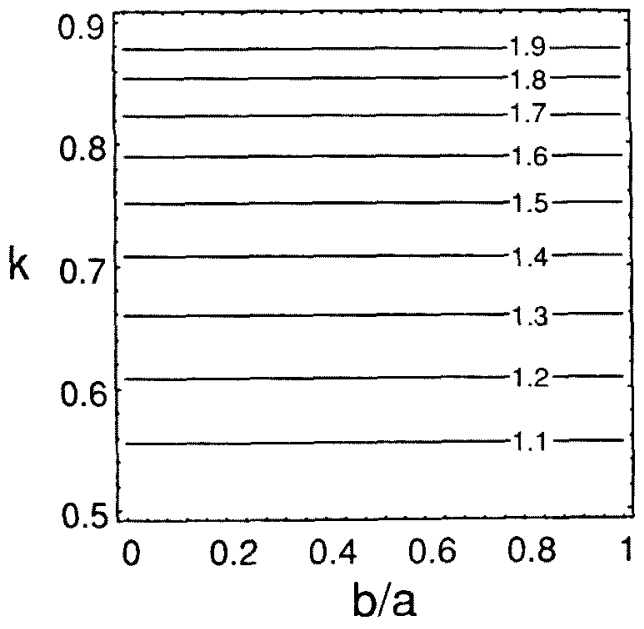

(b)

on the population dynamics, does not involve severe suppression, and is less vulnerable to uncertainty.

The comparison of its performance with that of a more conservative procedure, which is equivalent to Clark's, showed that the merit of the former procedure is not very large unless the intensity of interspecific competition is very high and a high target level is selected. Thus, the intentional depression below the target level may not be justified unless the existence of severe competition between species is demonstrated. Otherwise, depression to the target level may be sufficient even if we consider the recovery of the currently depressed species.

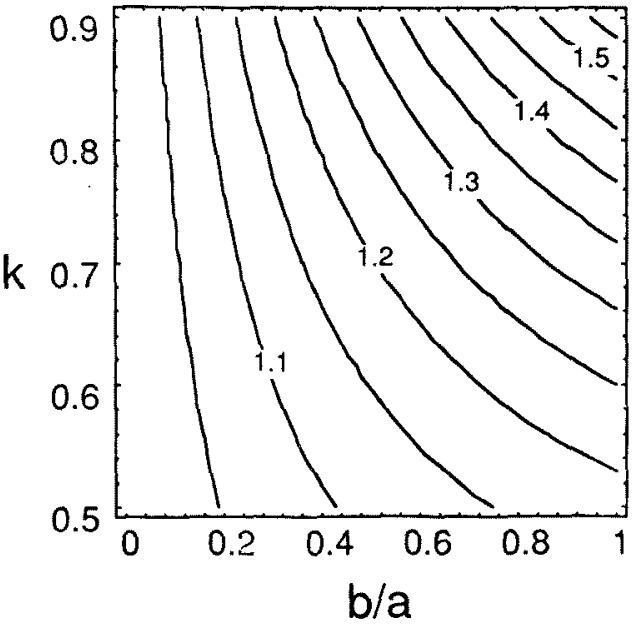

(c)

Fig. 6. Numerical example of $T_{1}, T_{2}$, and $\frac{T_{1}}{T_{2}}$.

The initial point and target is $\mathrm{A}\left(0.1 x^{*}, y_{\infty}\right)$ and $\mathrm{B}\left(x_{\infty}, y_{\infty}\right)=\left(k x^{*}, k y^{*}\right)$, respectively.

a) $T_{1}$ as a function of $\frac{b}{a}$ and $k . \quad T_{1}$ is standardized so that it takes 1 when $k=0.5$ and $b=0$. b) $T_{2}$ as a function of $\frac{b}{a}$ and $k . \quad T_{2}$ is standardized in the same manner as $T_{1}$. c) $\frac{T_{1}}{T_{2}}$ as a function of $\frac{b}{a}$ and $k$.

The effect of uncertainty in population dynamics may be analyzed by using a stochastic analogue of our model, and we may be able to obtain the optimal harvesting strategy in a tradeoff between the average recovery time and the risk of extinction. Such a stochastic analysis usually requires computer simulation of the individual situation and the result thus obtained will be strongly situation-dependent. However, it will be indispensable when the application of the procedure to a real fishery system is intended.

\section{Acknowledgements}

We thank Dr. H. Matsuda and two anonymous reviewers for helpful comments. This work is partly supported by a Japan Ministry of Education, Science and Culture Grant-inAid for Scientific Research on Priority Areas (\#319), Project "Symbiotic Biosphere: An Ecological Interaction Network Promoting the Coexistence of Many Species". 


\section{References}

1) K. Kawasaki: Sakana no Shigengaku, Otsukj-shoten, Tokyo, 1983, pp. 25-27.

2) International Whaling Commission: Report of the special meeting on southern hemisphere minke whales, Seattle, May, 1978. Rep. Int. Whal. Commn, 29, 349-358 (1979).

3) D. E. Gaskin: The Ecology of Whales and Dolphins, Heinemann Educational Books Ltd, London, 1982, pp. 318-324.

4) J. Harwood: The effects of inter-specific competition on the choice of management policies for fin and sei whales. Rep. Int. What Commn, 29, 167-169 (1979).

5) C.W. Clark: Mathematical Bioeconomics: The Optimal Management of Renewable Resources, 2nd ed. Wiley-Science, New York, 1990, pp. 168-196 and pp. 324-331.

6) K. J. Sainsbury: The ecological basis of multi-species fisheries, and management of a demersal fishery in tropical Ausatralia, in "Fish Population Dynamics" (ed. by J. G. Gulland), 2nd ed., John Wiley and Sons Ltd., Chichester, 1988, pp. $349-382$.

\section{Appendix 1}

The globally stable equilibrium of a general Lotka-Volterra competition system of two stocks

$$
\left\{\begin{array}{l}
\frac{\mathrm{d} x}{\mathrm{~d} t}=r_{x}(1-a x-b y) x, \\
\frac{\mathrm{d} y}{\mathrm{~d} t}=r_{y}(1-c x-d y) y,
\end{array}\right.
$$

exists if

$$
\frac{c}{a}<1<\frac{d}{b}
$$

and is

$$
\left\{\begin{array}{l}
x^{*}=\frac{d-b}{a d-b c}, \\
y^{*}=\frac{a-c}{a d-b c} .
\end{array}\right.
$$

(Assume that $r_{y}>r_{x}$ without loss of generality). On a straight line segment, $m$, connecting $(0,0)$ and $\left(x^{*}, y^{*}\right)$ defined by

$$
m:(x, y)=s\left(x^{*}, y^{*}\right) \quad(0<s<1),
$$

velocity of change in $x$ under no harvesting is

$$
\begin{aligned}
\frac{\mathrm{d} x}{\mathrm{~d} t} & =r_{x}\left(1-a s x^{*}-b s x^{*}\right) x^{*} \\
& =(1-s) r_{x} x^{*}
\end{aligned}
$$

and, similarly,

$$
\frac{\mathrm{d} y}{\mathrm{~d} t}=(1-s) r_{y} y^{*}
$$

This implies that

$$
\begin{aligned}
& \frac{\mathrm{d} y}{\mathrm{~d} x}=\frac{r_{y} y^{*}}{r_{z} x^{*}} \\
&>\frac{y^{*}}{x^{*}}
\end{aligned}
$$

when a no-harvesting trajectory intersects $m$. The inequality in (A1-2) indicates that the noharvesting trajectory intersects $m$ from below, which implies that a no-harvesting trajectory leading to the target, $l$, does not cross $m$ and lies below $m$. Thus, $y / x$ should be reduced lower than $y_{\infty} / x_{\infty}$, if the target is set so that the relative abundance of two stocks is not different from that at the virgin level.

On a no-harvesting trajectory,

$$
\frac{\mathrm{d} y}{\mathrm{~d} x}=\frac{r_{y}(1-c x-d y) y}{r_{x}(1-a x-b y) x},
$$

which is an increasing function of $r_{y} / r_{x}$. Thus $l$ lies lower as $r_{y} / r_{x}$ gets larger, which implies that $x$ should be reduced more severely as the difference in intrinsic growth rate gets larger.

When $B\left(x_{\infty}, y_{\infty}\right)$ lies below the line segment $m$, it can be easily shown in a similar way that, on a no-harvesting trajectory,

$$
\frac{\mathrm{d} y}{\mathrm{~d} x}>\frac{y}{x}
$$

is satisfied if $(x, y)$ lies below the line segment

$$
m^{\prime}: \quad(x, y)=s\left(x_{\infty}, y_{\infty}\right) \quad(0<s<1),
$$

Thus $l$ which leads to the target below $m$, lies below $m^{\prime}$. This indicates that the relative abundance of $y$ should be reduced below that at the target when $B$ lies below $m$.

\section{Appendix 2}

In this appendix, we obtain the time needed to attain the target in system (4) by each of the two paths shown in Fig. 5. The target and the initial conditions are

$$
\left(x_{\infty}, y_{\infty}\right)=k\left(x^{*}, y^{*}\right) \quad(0<k<1)
$$

and

$$
\left(x_{0}, y_{0}\right)=\left(x_{0}, y_{\infty}\right) \text {, }
$$

respectively. We assume $r_{x}<r_{y}, a>b$ and $x_{0}<x_{\infty}$. By defining $X=a x, Y=a y$, (4) reduces to

$$
\left\{\begin{array}{l}
\frac{\mathrm{d} X}{\mathrm{~d} t}=r_{x}(1-X-\beta Y) X-a h_{x}, \\
\frac{\mathrm{d} Y}{\mathrm{~d} t}=r_{y}(1-\beta X-Y) Y-a h_{y},
\end{array}\right.
$$

where $\beta=b / a$. Hereafter we analyze (A2-2) instead of (4), and we define $X_{\infty}=a x_{\infty}, Y_{\infty}=a y_{\infty}, X_{0}=$ $a x_{0}, Y_{0}=a y_{0}, X^{*}=a x^{*}$ and $Y^{*}=a y^{*}$. By (A2-1) and (A2-2)

$$
\left(X_{\infty}, Y_{\infty}\right)=\left(\frac{k}{1+\beta}, \frac{k}{1+\beta}\right)
$$


Thus on path 1,

$$
\frac{\mathrm{d} X}{\mathrm{~d} t}=r_{x}\left(1-X-\beta Y_{\infty}\right) X
$$

and the time needed to attain the target is

$$
\begin{aligned}
T_{1}= & \int_{X_{0}}^{X_{\infty}} \frac{1}{(d X / \mathrm{d} t)} \mathrm{d} X \\
= & \int_{X_{0}}^{X_{\infty}} \frac{1}{r_{x}\left(1-X-\beta Y_{\infty}\right) X} \mathrm{~d} X \\
= & \frac{1}{r_{x}\left(1-\beta X_{\infty}\right)}\left[\log \frac{X_{\infty}}{X_{0}}-\log \frac{(1+\beta) X_{\infty}-1}{\left(X_{0}+\beta X_{\infty}-1\right)}\right] \\
= & \frac{1}{r_{x}(1-\beta k /(1+\beta))}\left[\log \frac{k}{(1+\beta) X_{0}}\right. \\
& \left.-\log \frac{k-1}{\left(X_{0}+\beta k /(1+\beta)-1\right)}\right],
\end{aligned}
$$

which is equivalent to (5) in the main text.

On path 2,

$$
\frac{\mathrm{d} X}{\mathrm{~d} t}=r_{x}(1-X-\beta X) X
$$

after the relative abundance of two stocks reaches its final value. Thus, if we assume that the time needed to make the relative abundance at its final value is negligible, the time needed to attain the target is

$$
\begin{aligned}
T_{3} & =\int_{X_{0}}^{X_{\infty}} \frac{1}{(\mathrm{~d} X / \mathrm{d} t)} \mathrm{d} X \\
& =\int_{X_{0}}^{X_{\infty}} \frac{1}{r_{x}(1-X-\beta X) X} \mathrm{~d} X \\
& =\frac{1}{r_{x}}\left[\log \frac{X_{\infty}}{X_{0}}-\log \frac{(1+\beta) X_{\infty}-1}{(1+\beta) X_{0}-1}\right] \\
& =\frac{1}{r_{x}}\left[\log \frac{k}{(1+\beta) X_{0}}-\log \frac{k-1}{(1+\beta) X_{0}-1}\right],
\end{aligned}
$$

which is equivalent to (6) in the main text.

Both $T_{1}$ and $T_{2}$ are inversely proportional to $r_{x}$. If $X_{0}=k^{\prime} X^{*}$, as in the case considered in Fig. 6 ,

$$
T_{2}=\frac{1}{r_{x}}\left[\log \frac{k}{k^{\prime}}-\log \frac{k-1}{k^{\prime}-1}\right],
$$

which is independent of $\beta$. 